\title{
Hydrodynamics of Decretion Disks of Rapidly Rotating Stars
}

\author{
Petr Kurfürst \\ Department of Theoretical Physics and Astrophysics, Masaryk University \\ Kotlářská 2, CZ-611 37 Brno, Czech Republic \\ email: petrk@physics.muni.cz
}

\begin{abstract}
During the evolution of hot stars, the equatorial rotational velocity can approach its critical value. Further increase in rotation rate is not allowed, consequently mass and angular momentum loss is needed to keep the star near and below its critical rotation. The matter ejected from the equatorial surface forms the outflowing viscous decretion disk. Models of outflowing disks of hot stars have not yet been elaborated in detail, although it is clear that such disks can significantly influence the evolution of rapidly rotating stars. One of the most important features is the disk radial temperature variation because the results will help us to specify the mass and angular momentum loss of rotating stars via decretion disks.
\end{abstract}

Keywords. Rotation, mass loss, hydrodynamics

\section{Basic theoretical considerations}

In contrast to the usual stellar wind mass loss we study the role of mass loss via an equatorial outflowing viscous decretion disk evolution in massive stars (Krtička et al. 2011). Evolutionary contraction brings massive star to critical rotation: it leads to the formation of the disk. Further increase in rotation rate is not allowed $(\dot{\Omega}=0)$, net loss of angular momentum is given by $\dot{L}=\dot{I} \Omega_{\text {crit }}$, where $\Omega_{\text {crit }}=\sqrt{G M / R_{\text {eq }}^{3}}$ is the critical rotation frequency. The viscous coupling in a decretion disk can transport angular momentum outward to some outer disk radius, $R_{\text {out }}$. When a Keplerian disk is present, in comparison with the case where mass decouples in a spherical shell just at the surface of the star, the mass loss is then reduced by a factor

$$
\frac{3}{2} \sqrt{R_{\text {out }} / R_{\text {eq }}}
$$

Key point of the analysis: the angular momentum loss from the decretion disk can greatly exceed the angular momentum loss from the stellar wind outflow.

\section{Numerical approach}

For the numerical modelling, it is necessary to solve the system of hydrodynamic equations in cylindrical coordinates (Krtička et al. 2011). Except for the mass conservation (continuity) equation, we have to include the equations for stationary conservation of $R$ and $\phi$ components of momentum, supplemented by appropriate boundary conditions.

For further calculations, the Shakura-Sunyaev $\alpha$ viscosity parameter is introduced (Shakura \& Sunyaev 1973); it expresses the quantity $\tilde{v} / a$ (somewhat simplified since there are also effects of the magnetic field), where $a$ is the sound speed $\left(a^{2}=k T / \mu m_{H}\right)$ and we have $v_{R}\left(R_{\text {crit }}\right)=a$.

The temperature distribution in the radial direction is assumed as $T=T_{0}\left(R_{\mathrm{eq}} / r\right)^{p}$, where $p$ is a free parameter (power law). Some of recent models (e.g. Carcioffi et al. 

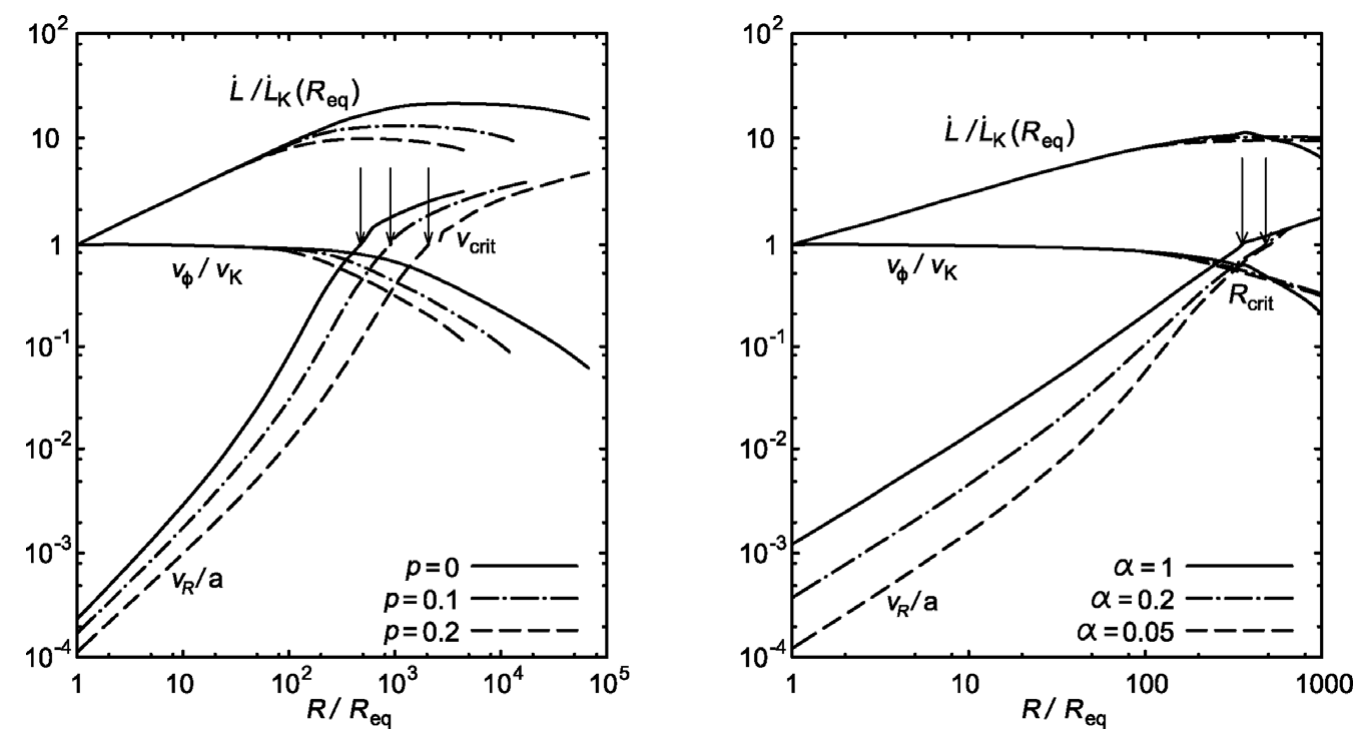

Figure 1. The graphs of dependence of relative radial and azimuthal velocity and relative angular momentum loss on radius.

2008) calculate the temperature distribution in the inner region of the disk as nearly isothermal $\left(T_{0}=\frac{1}{2} T_{\text {eff }}, p=0\right)$. But, for the calculations of the structure of outer parts of the disk, it is necessary to consider also the power law temperature decline $(p>0)$.

The system of three hydrodynamic equations (continuity equation, equations of stationary conservation of $R$ and $\phi$ components of momentum) is numerically approximated by differentiation at selected radial grid points with the use of the Newton-Raphson method (Krtička 2003).

\section{Results of numerical solution}

The selected stellar parameters are: spectral type B0, $T_{\text {eff }}=30000 \mathrm{~K}, M=14.5 M_{\odot}$, $R=5.8 R_{\odot}$ (Harmanec 1988). The left graph shows the dependencies of relative radial and azimuthal velocities and the relative loss of angular momentum on radius, the isothermal disk with various viscosity parameters $\alpha$ is considered $\left(T_{0}=\frac{1}{2} T_{\text {eff }}, p=0\right)$. At the critical radius, $R_{\text {crit }}: v_{R}=a$ (sonic point).

The right graph shows the same dependence for fixed viscosity parameter $\alpha=0.1$ and for different temperature profiles.

At large radii, the disk is not rotating as a Keplerian one; in the supersonic region $\left(v_{R}>a\right)$ the rotation velocity rapidly decreases as a consequence of the adopted $\alpha$ viscosity parameter. The increase of parameter $p$ (which means added cooling) implies an increase in the critical radius (the sonic point radius) and angular momentum loss. It will, therefore, be useful to calculate the models using different basic expressions for the viscous coupling.

\section{References}

Carciofi, A. C. \& Bjorkman, J. E. 2008, ApJ, 684, 1374

Harmanec, P. 1988, BAICz, 39, 329

Krtička, J. 2003, ASPC, 288, 259

Krtička, J., Owocki, S. P., \& Meynet, G. 2011, $A \& A$, 527, 84

Shakura, N. I. \& Sunyaev, R. A. 1973, A\&A, 24, 337 